POLSKA AKADEMIA NAUK Z ZAKEAD BADANIA SSAKOW

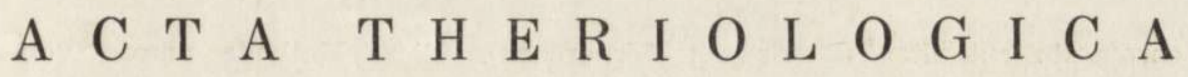
VOL. III, 9.

\title{
Cytologische Methode der Geschlechtsbestimmung bei Embryonen und das Geschlechtsverhältnis bei Fefus von Sorex araneus araneus $\mathbf{L}$. \\ Cytologiczna metoda określania płci zarodków i stosunek ilości samców do samic u plodów Sorex araneus araneus $\mathbf{L}$.
}

I. Einführung

II. Material und Methode

III. Veränderlichkeit der Gestalten und Masse der Gonaden . . 124

IV. Geschlechtsbestimmung des Embryos durch die cytologische Methode

V. Geschlechtsverhältnis bei Fetus von S. araneus . . . . 131

VI. Diskussion der Ergebnisse . . . . . . . . . 134

VII. Folgerungen 137

Schrifttum . . . . . . . . . . . . . . 138

Streszczenie

\section{EINFUHRUNG}

Die Geschlechtsbestimmung bei geschlechtsunreifen jedoch schon ausgewachsenen Spitzmäusen ist recht schwierig und bedingt in der Regel Seziereingriffe. Diese Schwierigkeiten vervielfachen sich noch bei Embryonen, bei welchen infolge der eigenartigen Entwicklung des Geschlechtsapparates anatomische Sezierungen keine positive Ergebnisse geben, aber des öfteren noch F'ehler herbeiführen. Bekanntlich kann man das Fetusgeschlecht, wenn sich dies anatomisch nicht durchführen lässt histologisch bestimmen oder cytologisch vermittels der Bestimmung der Zellkernanzahl, welche Geschlechts- 
chromatin enthalten (B a r r'sche Körperchen). Diese Methode wurde von Craham (1954, a, b), zur Geschlechtsbestimmung der Fetus bei den Katzen angewandt.

Der Zweck meiner Arbeit lag: a) in der Bearbeitung einer Methodik zur Geschlechtsbestimmung in den jeweiligen Würfen ${ }^{1}$ ) auf histologischem Wege; b) in der Feststellung, ob und in welchem Grade sich die cytologische $\mathrm{Me}$ thode zur Geschlechtsbestimmung eignen würde, um so mehr, als sie doch in der Anwendung als Leichtere gilt.

Ausserdem stellte ich mir als Aufgabe zu überprüfen, wie sich die Relation der Männchen den Weibchen gegenüber bei Embryonen gestaltet sowie das Ergebnis mit den im Freiland unter den erwachsenen Individuen vorherrschenden Verhältnissen zu vergleichen.

\section{MATERIAL UND METHODE}

Die Spitzmäuse wurden im Jahre 1954 im Białowieża - Nationalpark gefangen. Das Material ist im Besitz des Institutes für Säugetierforschung in Białowieża.

Um bei Sezierungen frühe Schwangerschaften nicht zu übersehen, nahm man nicht nur diejenigen Individuen, bei denen diese schon deutlich war, aber auch diejenigen, bei welchen der Uterus vergrössert war, was die Hoffnung aufkommen liess, dass sich diese Tiere in der Periode der frühen Implantation befinden könnten. Bei frühen Schwangerschaften (Fetus bis $2 \mathrm{~mm}$ ) fixierte man die Gebärtmutter gänzlich in der Flüssigkeit von Bou in; bei Fortgeschrittenen dagegen (Fetus über $2 \mathrm{~mm}$ Länge) präparierte man die Fetus heraus und fixierte sie dann in derselben Flüssigkeit. Vor dem Eingriff registrierte man die Fetusanzahl in jedem Gebärmutterhorn und bezeichnete ihre Lage mit nacheinander folgenden Nummern. Man benutzte dabei folgende Symbole: L - linkes, P - rechtes Horn. Die laufende Nummerierung begann man von der Seite des Eierstockes. Die Embryonen bewahrte man in $75 \%$ Alkohol auf.

Das Material hestand aus 38 schwangeren Weibchen, welche in der Zeitspanne von Mai bis September gefangen wurden.

Die Fetuszusammenstellung der einzelnen Weibchen war nicht immer damit übereinstimmend, was in der Kartotheke des betreffenden Tieres angegeben worden war.

Eine Übereinstimmung der Anschriften mit dem vorhandenen Material fand ich nur in 11 Fällen vor. Bei den übrigen fehlten je ein Embryo (20 Würfe), je zwei Embryonen (6 Würfe) und 3 Embryonen (1 Wurf). Dies waren Individuen die zu früheren Versuchszwecken verbraucht wurden.

In 14 Würfen bestimmte ich das Embryogeschlecht nur vermittels der cy-

1) Ich werde den Begriff „Wurf” zur Bestimmung eines Embryonen-Ensemble's vor der Geburt benützen, obwohl es allgemein üblich ist, diesen Begriff in Bezug auf schon geborene Individuen zu gebrauchen. 
tologischen Methode, in weiteren 15 - mit Hilfe der Cytologischen und der Histologischen. In 9 dagegen vermittels der Cytologischen, wobei jedoch einige auch histologisch bezeichnet wurden.

Falls Embryonen schon entwickelte Extremitätsanlagen hatten, führte ich die Kopfspitze-Aftermessung nach Godlewski (1950) durch (Entfernung zwischen der Kopfspitze durch die Mitte des Mesencephalons und der

\section{Tabelle 1.}

Gesamte Zusammenstellung der untersuchten Embryonen ${ }^{1}$ ).

\begin{tabular}{|c|c|c|c|c|c|}
\hline$M \circ n a t$ & Ma1 & Junt & Jul1 & August & September \\
\hline 1 & $\begin{array}{r}18 / 7 \\
17 / 7 \\
17 / 6 \\
110 / 9\end{array}$ & $\begin{array}{ll}18 / 7 \\
17 / 5 \\
18 / 8 \\
18 / 8 \\
14 / 2\end{array}$ & $\begin{array}{l}17 / 5 \\
19 / 9 \\
17 / 7 \\
16 / 5 \\
1515 \\
16 / 4\end{array}$ & $\begin{array}{ll}16 / & 6 \\
17 / & 6 \\
16 / & 6 \\
16 / & 5 \\
14 / & 3 \\
14 / & 3 \\
15 / & 4 \\
16 / & 5 \\
15 / & 5\end{array}$ & $\begin{array}{l}15 / 4 \\
16 / 3 \\
16 / 5 \\
14 / 4 \\
14 / 3 \\
14 / 3 \\
17 / 6 \\
13 / 2 \\
15 / 3 \\
15 / 4 \\
14 / 3 \\
18 / 2 \\
121\end{array}$ \\
\hline Zusammen & $132 / 29$ & 135130 & $140 / 35$ & $149 / 43$ & $163 / 48$ \\
\hline
\end{tabular}

1) In Klammern -- Gesamtzahl der Embryonen.

Schwanzkrümmung des Embryos.). Bei Embryonen die kleiner waren als von 1,8 bis $2,0 \mathrm{~mm}$, also solche die noch keine entwickelten Gliedmassenanlagen hatten, führte ich die sogenannte Messung der grössten Länge nach Go dlewski durch (Entfernung zwischen zwei am weitesten entlegenen Punkten des Embryokörpers). Natürlich ist jede solche Messung ungenau. Sie ermöglicht jedoch eine gewisse Klassifikation des Materiales und eine angehende Altersbestimmung des Embryo's.

Nach der Messung spannte ich mit Hilfe von entomologischen Stecknadeln die Embryonen auf einem Wachsplättchen auf und präparierte sie unter dem Binokular mit einem Skalpell, das aus einem sehr dünnen und kleinen Bruchstück einer Rasierklinge hergestellt wurde (in einer Klemmpinzette befestigt). Solch ein Skalpelltyp ist bei der Arbeit unbedingt bequemer zu handhaben als das beste okulistische Skalpell.

Ich schnitt die Ha!ıthüilien quer durch den Bauch etwas unterhalb des Nabels durch, und nachdem bog ich sie etwas nach hinten ab. Auf solche Weise kann man diejenige Gegend sichtbar werden lassen, in der Nieren und Gonaden liegen. Bei der Präparation ist es sehr wichtig, den Bauch unterhalb des Nabels aufzuschneiden, denn im widrigen Falle hebt sich mit der geho- 
benen Bauchwand ebenfalls der Darm, und es entstehen unnütze Schwierigkeiten bei dem Aufsuchen der Gonaden und ihrem Erreichen.

Ich habe es schon erwähnt, dass man aus dem oberflächlichen Bild des Geschlechtsapparates keine richtige Geschlechtsbestimmung des Fetus erhalten kann; sogar nicht bei den ältesten Embryonen, über welche ich verfügte (13,5 mm Länge). Ein Beweis hierfür ist die histologische Kontrolle der Bestimmungen auf Grund der äusserlichen Morphologie, die erwiesen hatte, dass man nur ein einziges Mal auf 20 Bestimmungen also zufällig das Geschlecht richtig getroffen hatte. Bei anatomischen Proben der Geschlechtsbestimmung wurden die Gonaden sehr genau in einer bestimmten und immer gleichen Skala aufgezeichnet. Eingehende Vergleichungen der Zeichnungen führten ebenfalls zu keinem positiven Ergebnis. Die Schwierigkeiten ergeben sich daraus, dass die Krümmungen der Leitungen, die die Angehörigkeit zu dem einen oder dem anderen Geschlecht suggerieren, einen ganz zufälligen Charakter haben. Es ist möglich, dass dies, nur Artefakten sind, die beim Fixieren entstanden sind. Ausserdem umgibt der Eileiter auf eine solche Weise den Eierstock, dass man ihn von dem Nebenhoden, der den Hoden halbkreisförmig umfasst, nicht unterscheiden kann. Eine ähnliche Lage des Eileiters und des Nebenhodens finden wir bei ausgewachsenen aber noch geschlechtsunreifen Individuen vor. Dort erinnert jedoch die Gestalt der Gebärmutter ganz bestimmt an den Buchstaben „T" im Gegensatz von den Samenleitern bei Männchen, die sich in Gestalt des Buchstaben „V” legen.

Die Gonaden nahm ich heraus und mass ihre lange und kurze Achse. $10 \mu$ Parafinschnitte färbte ich mit Hämatoxylin nach $\mathrm{Ehrlich}$.

Bei Embryonen die kleiner waren als 6,5-7,0 mm, war das Auspräparie ren der Gonaden sehr schwierig, denn sie unterlagen meistenteils während der Präparierung der Vernichtung oder der Beschädigung. Dabei verkrümelte sich das Embryo in diesem Stadium bei der Berührung mit dem Skalpell leicht, also war der Eintritt in die Bauchhöhle doch recht peinlich.

In solchen Fällen schnitt ich das Fetusgliedmass ab. Parafinschnitte von einer $10 \mu$ Stärke färbte ich mit Hämatoxylin nach $\mathrm{Ehrlich}$, und bestimmte dann das Geschlecht vermittels der cytologischen Methode.

Sehr kleine Embryonen, bei denen die Gliedmassen noch nicht sichtbar wurden, schnitt ich durch ihr Ganzes und das Geschlecht bestimmte ich durch Geschlechtschromatin.

\section{VERÄNDERLICHKEIT DER GESTALTEN UND MASSE DER GONADEN}

Während der Untersuchungen stellte ich fest, dass die Gonade während der Entwicklung (dies betrifft beide Geschlechter im gleichen Grade) auf eine charakteristische Weise ihre Gestalt mit dem Alter (zunehmendem) ändert. Bei jüngeren Embryonen mit Körperlänge von 6,5 bis $8,0 \mathrm{~mm}$ sind die Gonaden verlängert und im Verhältnis zum Körper gross. In der Fetuslebensperiode unterliegt ihre Länge fast keiner Veränderung, obwohl die Embryokörperlänge von 
$6 \mathrm{~mm}$ bis $14 \mathrm{~mm}$ anwächst (d. i. über $100 \%$.) In der frühen Periode ihrer Entwicklung sind die Gonaden noch nicht mit den Ligamenten verbunden, sondern liegen vielmehr lose in der Bauchhöhle (Abb. 1 und 2). Das Bild der Veränderlichkeit von Gestalten und Massen der Gonaden ist auf Tabelle 2 dargestellt. Die Länge (Mittelgrösse) der Gonaden oder der Eierstöcke beträgt bei den Jüngsten $0,44 \mathrm{~mm}$; die Breite $0,17 \mathrm{~mm}$. In der zweiten Wachstumsgruppe (Embryonen von 8-10 $\mathrm{mm}$ Länge) erreicht die Gonadenbreite im

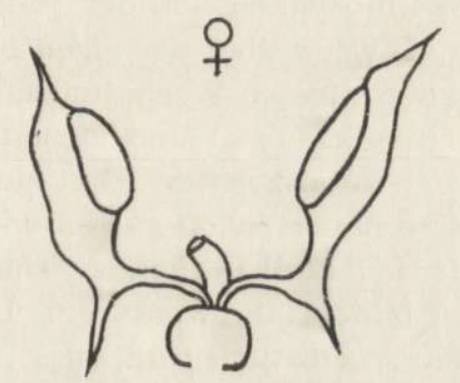

Abb. 1. Geschlechtsapparat des Embryos (Nr. 5938, L2, ᄋ ) 8,2 mm Länge. Vergr. z-a $80 \times$.

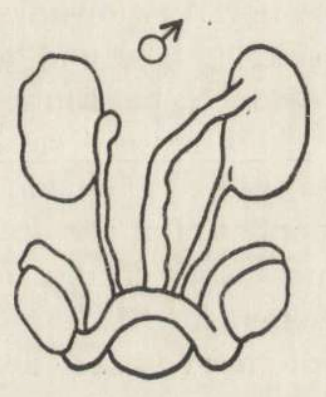

Abb. 2. Gonadenanlage beim Embryo (Nr. 64951, đో) $10,9 \mathrm{~mm}$ Länge. Vergr. $\mathrm{z}-\mathrm{a} 24 \times$.

\section{Tabelle 2 .}

Veränderlichkeit der Gestalten und Masse von Gonaden der Embryonen und der erwachsenen Spitzmäuse.

\begin{tabular}{|c|c|c|c|c|c|c|}
\hline Alter & $\begin{array}{l}\text { K. }+ \text { R-Iänge } \\
\text { In } r=1 \text {. }\end{array}$ & $\mathrm{n}$ & $\begin{array}{c}\text { Gonadenmasse } \\
\text { 1n mm. }\end{array}$ & \begin{tabular}{|c|} 
Anze1ger \\
der \\
Gonaden- \\
gestaltung \\
$2 /$
\end{tabular} & $\frac{a \cdot b \cdot \pi}{4}$ & $\begin{array}{l}\text { Grössen- } \\
\text { anze1ger } \\
\text { der } \\
\text { Gonaden 1m } \\
\text { Verha1tn1s } \\
\text { zur } \mathbb{R} \text {. + R. }\end{array}$ \\
\hline \multirow{3}{*}{ Bmbryone } & $6-8$ & 21 & $0.44 \times 0.17$ & 2.59 & 0.39 & 15.90 \\
\hline & $9-10$ & 37 & $0.45 \times 0.22$ & 2.05 & 0.41 & 20.00 \\
\hline & $11-14$ & 22 & $0.43 \times 0.25$ & 1.72 & 0.45 & 27.90 \\
\hline $\begin{array}{l}\text { Junge aus dem } \\
\text { Jun1, } \\
\text { gesohlechts- } \\
\text { unre1f }\end{array}$ & 60.4 & 20 & $2.2 \times 1.3$ & 1.69 & 2.24 & 27.45 \\
\hline $\begin{array}{l}\text { Uberwinter- } \\
\text { linge aus dem } \\
\text { August }\end{array}$ & 72.8 & 20 & $9.1 \times 5.7$ & 1.59 & 40.80 & 8.00 \\
\hline
\end{tabular}

1) Es sind hier Mittelmasse der Gonaden für diese Altersklasse angegeben worden.

2) Gonadenlänge geteilt durch Gonadenbreite. a - Gonadenlänge; b - Gonadenbreite. 
Mittelwert $0,22 \mathrm{~mm}$, schliesslich bei den ältesten Embryonen beträgt sie bis $0,25 \mathrm{~mm}$, folgedessen bildet sich die Gonade während der Periode des Fetuslebens um und wird aus einer deutlich verlängerten und elipsoidalen fast zu einer kugligen Form. Diese Veränderungen illustriert die Veränderlichkeit des Anzeigers $\frac{\text { Gonadenlänge }}{\text { Gonadenbreite }}$ die von $0,25-0,17$ schwankt.

Veränderungen beobachtet man ebenfalls in der Gestaltung u. Grösse der Ausführungswege. Bei Embryonen aus der jüngsten Gruppe sind sie lang und dominieren als wenn über der eigentlichen Gonade (Abb. 1), bei Embryonen aus der ältesten Wachstumsgruppe dagegen (Abb. 2) sind sie kürzer mehr gedrungen und ziehen sich im Verhältnis zur Gonade als wenn auf den zweiten Plan zurück. Embryonengonaden aus diesem Stadium haben schon gut entwickelte Ligamenta, durch die sie mit dem Bauchfell verbunden sind.

Embryonen im Stadium 8-10 mm bilden als wenn ein Übergangsstadium zwischen den extremen Wachstumsgruppen.

\section{GESCHLECHTSBESTIMMUNG DES EMBRYO'S DURCH DIE CYTOLOGISCHE METHODE}

B a r r stellte im Jahre 1950 fest, dass in den Zellkernen des Nervengewebes der Katze in der Nähe des Kernchens sich charakteristische Chromatinkörperchen von runder Gestalt befinden. Bei Männchen traten diese Gebilde nur in etlichen Kernzellen auf. $\mathrm{B}$ a r $\mathrm{r}$ ist der Ansicht, dass das Geschlechtschromatin aus der Verbindung von zwei heterochromatischen Teilen des Chromosomes $\mathrm{X}$ entsteht. Infolgedessen vermutet er, dass hier eine grössere Chromatinmasse im Vergleich mit dem Komplex der Geschlechtschromosomen XY produziert wird. Das Geschlechtschromatin aus den Zellkernen des Nervengewebes unterscheidet sich etwas von dem Chromatin, das in Zellkernen anderer Gewebe vorgefunden wird.

$\mathrm{B}$ a $\mathrm{r} \mathrm{r}$ beschrieb ebenfalls das Geschlechtschromatin, das in den Zellkernen der Epidermis vorgefunden wurde. Er gibt folgendes an²) „Das ist ein gewöhnlicher, einzelner Körper von zirka $1 \mu$ Durchmesser, der gleich hinter der inneren Fläche der Kernmembran gelegen ist.

Das Geschlechtschromatin kann nur dann identifiziert werden, wenn es sich auf den Peripherien des optischen Kernausschnittes

2) Zitat aus dem Englischen übersetzt. 
befindet. Von anderen Chromatinteilen kann man es auf Grund seines grösseren Ausmasses, seiner Gestalt und seiner Lage an der Kernmembran unterscheiden. Es ist schwere es mit dem Kernchen zu verwechseln, denn das Letztere ist grösser, von sphärischer Gestalt und ist in einer gewissen Entfernung von der Kernmembran gelegen".

Bisher entdeckte man das Geschlechtschromatin bei Primates, Artiodactyla und Carnivora. Bei Rodentia erwies sich die Geschlechtsbestimmung mit dieser Methode als unmöglich. $\mathrm{M}$ o o r e und $\mathrm{B}$ a r $\mathrm{r}$ hatten nämlich festgestellt, dass bei diesen Tieren zahlreiche, perinucleare Chromatinmassen in den Kernen der Neuronen beiderlei Geschlechts vorhanden sind, was die Entdeckung irgendeines Unterschiedes zwischen den männlichen und weiblichen Zellkernen unmöglich macht.

Wie ich es im Schrifttum feststellen konnte, hat man bisher keine Untersuchungen über das Geschlechtschromatin bei Insectivora durchgeführt, aber auf keinen Fall bei den Soricidae. Im Zusammenhang damit überprüfte ich, wie sich die Einteilung des Geschlechtschromatins in den Zellkernen bei den erwachsenen Spitzmäusen und Embryonen verhält. Bei den Letzteren war es natürlich notwendig, die erhaltenen cytologischen Bestimmungen vermittels der histologischen Methode zu kontrollieren.

Die Kontrollbestimmungen führte ich auf Embryonen von $7 \mathrm{~mm}$ Länge bei 15 Weibchen durch.

Das mikroskopische Bild des Hodenquerschnittes (Phot. 1) verändert sich in Abhängigkeit vom Alter recht wenig. Auf dem Querschnitt sieht man die Tunic a al bugine a und den zentralen Teil, der ein Querschnitt durch die Samenkanälchen ist. Die Hülle besteht aus einigen Zellschichten mit flachen und langgestreckten Kernen, die eng aneinander gelegen sind. Den zentralen Teil bildet eine Anhäufung von Zellen kugliger oder ovaler Gestalt, die die Anlagen der Samenkanälchen darstellen. Die Zellen sind klein und haben ovale Zellkerne. In der Embryoserie, derer ich mich bedient habe, bemerkte ich keine grösseren Unterschiede im Bilde des Hodenquerschnittes in Abhängigkeit vom Embryoalter. Man kann nur behaupten, dass die Hülle bei älteren Embryonen einer Verengung unterliegt.

Auf dem Eierstockquerschnitt von einem Embryo mit $7 \mathrm{~mm}$ Länge kann man drei konzentrisch gelegene Schichten unter- 
scheiden. Die am meisten nach inner liegende' Schicht ist kompakt und besteht aus Zellen mit länglichen und ovalen Kernen. Die Mittelschicht besteht aus locker anliegenden Zellen mit runden Kernen. Die dritte äusserliche Schicht bildet ein einschichtiges Epithelium, das aus Zellen vom Typ des Pflasterepitheliums besteht, die jedoch verhältnismässig hoch sind und eng aneinander liegen. Bei älteren Embryonen (Phot. 2) bleibt der Markteil gleich kompakt, aber im Rindenteil erfolgt eine grössere Lockerung des Gewebes. Die Zellkerne sind kleiner und färben sich teilweise dunkel. Solcher Kerne gibt es immer mehr, bis sie schliesslich fast den ganzen Rindenteil des Eierstockes einnehmen und nur hier und da treten Zellen mit kugligen Kernen auf. Das Epithelium ist immer noch einschichtig, hoch und mit eng aneinanderliegenden Zellen (Phot. 3).

Diese Unterschiede ermöglichen es schon auf den ersten Blick das Embryogeschlecht $\mathrm{zu}$ bestimmen. Ich habe festgestellt, dass bei Sorex araneus L., also beim typischen Vertreter der Familie der Soricidae, das Geschlechtschromatin sehr gut unterschieden werden kann. Sein Aussehen stimmt mit der Beschreibung von $B$ a r r gänzlich überein. Mann kann nur zusätzlich hinzufügen, dass es ein etwas mondsichelförmiges Gebilde ist, das an die Kernmembran anliegt und sich meistenteils stärker färbt als der Rest des Kernes (Phot. 4).

$\mathrm{Da}$ nun die Festsetzung aller Zahlenverhältnisse so ,auf das Auge" immer subjektiv ist, zählte ich 100 Zellkerne im Gesichtsfeld des Mikroskopes und in einem solchen Masstabe bestimmte ich die Kerne, die Geschlechtschromatin besitzen.

Ich stellte fest, dass es bei erwachsenen Weibchen durchschnittlich 33 Zellkerne mit Geschlechtschromatin auf die Gesamtzahl von 100 Zellkernen gibt und bei Männchen nur 8 Zellkerne mit Geschchlechtschromatin. Gewiss ist die von mir hier angegebene Durchschnittsanzahl, die die maximale oder minimale Frequenz des Auftretens von Zellkernen darstellt, nich ganz genau, denn ich untersuchte nur 8 erwachsene Individuen (darunter 4 Weibchen und 4 Männchen). Es ging mir hier vielmehr um die Festellung, ob sich bei erwachsenen Individuen die Anzahl der Zellkerne mit Geschlechtschromatin bei Weibchen und bei Männchen ähnlich verhält wie bei Embryonen.

Bei älteren Embryonen mit einer Körperlänge von 6,5 bis 14 $\mathrm{mm}$ ist das cytologische Bild klar und es darf kein Zweifel auf- 
kommen (das Material wurde auch histologisch kontrolliert). Die Anzahl der Zellkerne mit Geschlechtschromatin beträgt bei Weibchen 33 bis 51 (durchschnittlich 40) auf 100. Bei Männchen haben wir dagegen 3 bis 11 (durchschnittlich 7) Kerne mit Geschlechtschromatin auf 100 .

Tabelle 3.

Anzahl der Zellkerne, die Geschlechtschromatin besitzen, bei erwachsenen Individuen und Embryonen von Sorex araneus araneus $\mathrm{L}$.

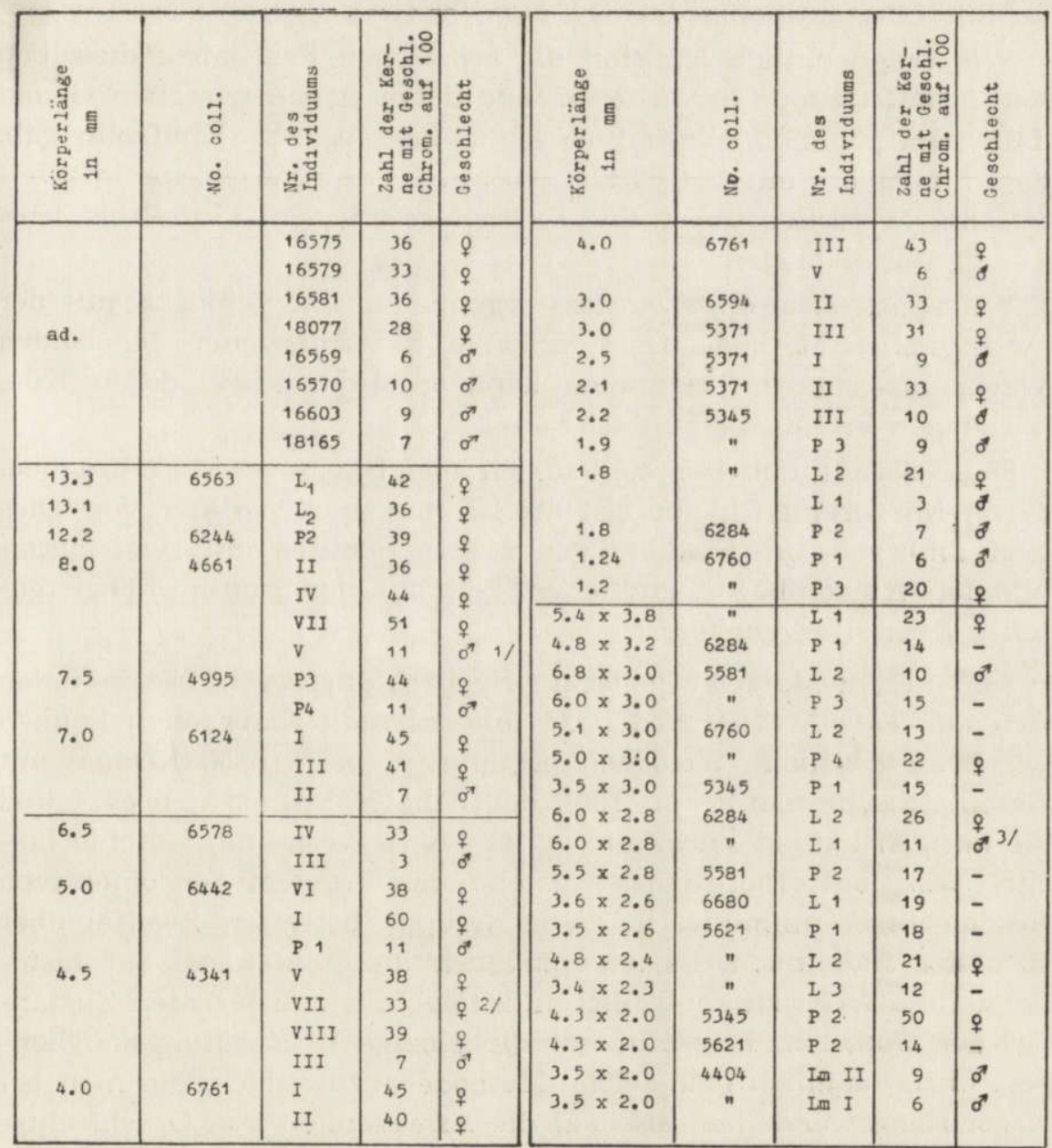

Embryone bei denen das Geschlecht bestimmte man: 1/ - Histologisch und cytologisch; 2/ - Nur cytologisch; $3 j$ - Embryonen kleiner als $1.8 \mathrm{~mm}$ Länge. Die hier angegebenen Masse sind Breiten- u. Längenmasse der Hervorhebung der Gebärmutter samt. Embryo. Leider, das Geschlecht unbestimmt. 
Die hier vorherschenden Verhältnisse sind denjenigen ähnlich, die wir bei erwachsenen Individuen angetroffen haben (Siehe Tabelle 3 ).

In der zweiten Gruppe der cytologich untersuchten Embryonen befinden sich jüngere Fetus mit Ausmassen 1,8-6,5 mm.

Bei diesen Embryonen können wir 2 deutliche Gruppen unterscheiden. Eine bei der das Geschlechtschromatin sich in den Kernen im Verhältnis - 21 - 60:100 vorfindet und die zweite Gruppe mit Chromatinanzahl 3-11 auf 100 Kerne.

Wie es ersichtlich ist, sind die erhaltenen Ergebnisse dieselben wie in der Gruppe der Älteren, und daher haben wir allen Grund dafür anzunehmen, obwohl es keine histologische Kontrolle gab, dass Exemplare mit 21-60 Kernen mit Geschlechtschromatin zweifelsohne Weibchen waren und Exemplare mit dem Verhältnis 3 bis 11 auf 100 Männchen.

Wir sehen also, dass wir fast regelmässig und fehlerlos mit der cytologischen Methode das Geschlecht bei Embryonen der beiden Wachstumsgruppen bestimmen können, also solcher, deren Körperlänge von $1,8-14 \mathrm{~mm}$ schwankt.

Diese Angelegenheiten stellen sich aber bei jüngeren Embryonen, deren Körperlänge kleiner ist als $1,8 \mathrm{~mm}$, nicht so klar vor. Dies sind Embryone im Stadium von 14-16 Somiten mit zwei ausgebildeten Kiemenbögen und einer fast auf der ganzen Länge geschlossenen Nervenrinne.

Auf diesem Material gemachte Beobachtungsergebnisse befinden sich auf Tabelle 3 . Von den 21 untersuchten Embryonen können wir 5 als deutliche Weibchen bestimmen (bei 21-50 Kernen mit Geschlechtschromatin auf 100), deutliche Männchen gab es 4 und die übrigen Individuen haben von 12 bis 19 Kerne mit Geschlechtschromatin auf 100; daher ensteht das Problem der objektiven Geschlechtsbestimmung. In dieser Gruppe betreffen Zweifel über $40 \%$ der Individuen. Es ist unmöglich dieses Material auf histoliogischem Wege zu kontrollieren, infolge des indifferenten Zustandes der Gonaden. Es bleibt nur die einzige Überprüfungsmöglichkeit übrig, nämlich eine solche Methode anzuwenden, die man bei Nagern angewandt hat, also auf die Anwesenheit von Geschlechtschromosomen in den Geschlechtszellen. Die Untersuchung dessen würde auf dem erwähnten Material ungemein schwierig sein, wenn nicht ganz unmöglich im Hinblick auf die Fixierungsart. 


\section{GESCHLECHTSVERHÄLTNIS BEI FETUS VON S. ARANEUS}

Dies Problem hat eine wesentliche Bedeutung, denn es ermöglicht den Mechanismus der Anzahlregulation des Geschlechtes festzustellen. Bekanntlich treffen wir bei vielen Säugergruppen bei Erwachsenen ein Verhältnis der Gechlechtsanzahl, das von dem traditionellen Schema 1:1 abweicht.

In meiner Arbeit war das Material, über das ich verfügte, nicht so gross, um sichere und gänzlich objektive Folgerungen ziehen zu können. Dennoch sind die Verhältnisse, die ich beobachtet habe, interessant und der Besprechung wert.

Tabelle 4.

Geschlechtsverhältnisse in den Würfen von Sorex araneus araneus L. in Abhängigkeit von den Jahreszeiten.

\begin{tabular}{|l|c|c|c|c|c|}
\hline NOn a t & Ma1 & Jun1 & Jul1 & August & September \\
\hline $\begin{array}{c}\text { Gesohlechts- } \\
\text { verhältn1s } \\
87: 99\end{array}$ & $16: 13$ & $20: 10$ & $21: 14$ & $19: 24$ & $30: 25$ \\
\hline $\begin{array}{c}\text { Anzahl der } \\
\text { Mrirfen }\end{array}$ & 4 & 5 & 6 & 9 & 14 \\
\hline
\end{tabular}

Zusammenstellungen von Zahlangaben des Geschlechtsverhältnisses sind auf Tabelle 4 dargestellt. Analisiert wurde eine volle Zusammensetzung des Materiales (190 Embryonen). Allgemein genommen, hatte ich 84 Weibchen und 106 Männchen. Wir haben also im Material ein deutliches Übergewicht von Männchen (58,8\%). Wenn wir jedoch die einzelnen Würfe durchanalysieren, so können sich die Verhältnisse verschieden gestalten. Bei der grössten Anzahl der Würfe traf ich kleine Zahlenunterschiede der Weibchen und Männchen. (Der Unterschied machte ein bis zwei Embryonen aus). Es gab aber auch Würfe, bei denen es eine Mehrheit an Weibchen oder Männchen gab. Man traf sogar solche ( 7 Fälle), die nur aus Männchen oder nur Weibchen bestanden.

Wir beobachten bei Fetus während aller Monate ausser August eine Überzahl an Männchen, und nur in dem letzten Monate haben wir eine Überzahl an Weibchen (Tabelle 4). Dieser Monat ist im Material verhältnismässig zahlreich repräsentiert, so dass man den Eindruck hat, dass wir hier mit einer objektiven Erscheinung zu tun haben. 
In der Arbeit von Borowski und Dehnel (1952) ist das zahlenmässige Verhältnis der Männchen zu den Weibchen (noch geschlechtsunreifen Individuen), angegeben worden, die in der Sommerperiode der Jahren 1949-1952 gefangen wurden.

Sofern die von den oben erwähnten Autoren erhaltenen Ergebnisse aus den Jahren 1951-1952 einen gewissen Vorbehalt wecken können, da das für diesen Zweck benutzte Material zu klein war, so stützen sich die Ergebnisse aus den Jahren 1949 i 1950 auf eine genügend grosse Anzahl der Beobachtungen. (Über 1300 in dem einen Jahr und 340 in dem andern). In beiden Jahren summiert, erwiesen die Autoren eine zahlenmässige Überlegenheit der Weibchen, und stellten insofern fest, dass in einer im Freiland lebenden Population die Verhältnisse in den konkreten Jahren sich umgekehrt gestalten können, als dies sich aus meinen Beobachtungen bei Embryonen ergibt. Beobachtungen anderer Autoren weisen bei Erwachsenen auf die Überlegenheit der Männchen hin und des öfteren auf eine sehr Grosse. Meiner Ansicht nach sind diese Beobachtungen nicht vollwertig, denn das Material war nicht auf Jungtiere und Uberwinterlinge eingeteilt. Wenn es sich um die Letzten handelt, können wir mit Gewissheit feststellen, dass, infolge der eigenartigen Form der Aktivität bei beiderlei Geschlecht, wir im Frühling und Sommer in den Fängen eine grosse ja sogar in gewissen Monaten eine sehr grosse Überlegenheit der Männchen erhalten können.

Es scheint mir, dass die von P u c e k (1959) zusammengestellten Angaben einen objektiven Charakter haben können. Er erwies, dass man in einer Zeitspanne von 10 Jahren unter den jungen Individuen eine Überzahl an Männchen (natürlich summarisch) festgestellt hat, obwohl sich die Verhältnisse in den einzelnen Jahren verschieden gestalten können.

Nicht weniger interessant ist, dass die zahlenmässigen Verhältnisse bei beiderlei Geschlecht sich in den einzelnen Monaten ändern können.

Aus der Arbeit von Borowski und Dehnel geht hervor, dass es in den Jahren 1949 und 1950 während aller sommerlichen Monate ausser dem Juni eine Uberlegenheit an Weibchen gab, im Juni aber an Männchen. Aus den Beobachtungen von $\mathrm{Pucek}$ ergibt es sich, dass während 10 Jahren bei Jungen immer im Juni eine Überzahl an Männchen war. In den anderen Monaten konnten 
sich diese Verhältisse verschieden gestalten, aber im August trat immer eine Überlegenheit an Weibchen auf. Es scheint interessant zu sein, dass ich unter den Fetus im August ebenfalls eine Überzahl an Weibchen hatte.

Schon auf Grund meines so geringen Fetusmateriales, muss man annehmen, was schliesslich von den in der Literatur angenommenen Anschaungen über dieses Problem nicht abschweift, dass hie:

Tabelle 5.

Geschlechtsverhältnis in den Würfen in Abhängigkeit von der Embryosterblichkeit.

\begin{tabular}{|c|c|c|c|c|c|c|}
\hline $\begin{array}{l}\text { Geschlechts- } \\
\text { verhältn1s }\end{array}$ & $\begin{array}{r}\text { Würe ohne } \\
\text { Sterblichke1t }\end{array}$ & 1 & 2 & 3 & 4 & 5 \\
\hline \multirow{8}{*}{$: 99$} & $76: 53$ & $12: 10$ & $11: 9$ & $6: 9$ & $0: 2$ & $1: 1$ \\
\hline
\end{tabular}

Tabelle 6.

Geschlechtsverhältnis in den Würfen in Abhängigkeit von der Anzahì der ovulierten Eier.

\begin{tabular}{|c|c|c|c|c|c|c|c|}
\hline $\begin{array}{c}\text { Anzahl der } \\
\text { ovulierten B1er }\end{array}$ & 4 & 5 & 6 & 7 & 8 & 9 & 10 \\
\hline $\begin{array}{c}\text { Gesohlechtsver- } \\
\text { hältn1s } \\
80: \$ 9\end{array}$ & $2: 1$ & $7: 6$ & $26: 27$ & $25: 18$ & $29: 17$ & $13: 10$ & $4: 5$ \\
\hline
\end{tabular}

eine eigenartige zahlenmässige Regelung des Geschlechtsverhältnisses erfolgt. Sie kann theoretisch genommen in den Perioden des Fetuslebens, des Nestlebens und des Aussernests-Lebens erfolgen. (Hier interessiert uns nur die Periode des Fetuslebens). Auf Tabelle 5 sind die zahlenmässigen Geschlechtsverhältnisse in denjenigen Würfen dargestellt, in denen man d. s. g. Fetussterblichkeit beobachtet hatte, aber auch solche in denen die Sterblichkeit nicht beobachtet wurde. Die Angeben zu dieser Tabelle ergänzte ich aus der Arbeit von Tarkowski (1957). In denjenigen Würfen in denen es keine Sterblichkeit gab, also wo sich der ursprüngliche Zustand erhielt, bestand eine grosse Überlegenheit an Männchen. Das Geschlechtsverhältnis beträgt 76 Männchen zu 53 Weibchen. In den weiteren Rubriken sind Geschlechtsverhältnisse in denjenigen Würfen angegeben worden, wo abgestorbene Embryone vorhanden waren. Man kann feststellen, dass das Geschlechtsverhältnis sich dort verändert hat und sich wie $1: 1-(30$ 
Männchen zu 31 Weibchen) verhâlt. Daraus könnte man schliessen, dass die Embryosterblichkeit die Geschlechtsverhältnisse regelt und die Anzahl der Männchen vermindert. Wie es Tarkowski (1957) festgestellt hat, tritt die Embryosterblichkeit vor allem im August und September auf, also würden es diese Monate sein, in denen die Regelung in maximaler Intensität auftritt.

Ich versuchte es festzustellen, ob das Geschlechtsverhältnis von der Wurfanzahl abhängig ist. Auf Tabelle 6 stellte ich die die Geschlechtsverhältnisse charakterisierende Anzahl der ovulierten Eier zusammen. (Ergänzende Angeben aus der Arbeit von $\mathrm{T}$ a r k ow ski). Zahlen, die ich angegeben habe, würden wohl, wie es mir scheint, darauf hinweisen, dass es keine Abhängigkeit zwischen der Weibchen- und Männchenanzahl und der Zahlenmässigkeit der Würfe gibt.

\section{DISKUSSION DER ERGEBNISSE}

Aus dem dargestellten Material geht hervor, dass die erhaltenen Effekte keine Entwicklung von weitgehenden Folgerungen erlauben. Der Grund hierfür liegt vor allem in dem kleinen mir zur Verfügung stehenden Material und das zusätzlich nur noch aus einer Saison stammte. Nichtsdestoweniger erhielt man gewisse konkrete Tatsachen und zwar: Man stellte bei Sorex araneus die Möglichkeit der Aussonderung von Geschlechtschromatin fest. Weiterhin bearbeitete man vermittels dessen Hilfe die Methode der Geschlechtsbestimmung der Individuen. Ferner stellte man die interessante Tatsache fest, dass das für beide Geschlechter typische und charakteristische Verhältnis der Kerne, die Geschlechtschromatin besitzen zu denjenigen die es nicht besitzen hier nur scheinbar eine gewisse und verhältnismässig ziemlich späte Periode des Embryo-Lebens verpflichtet und zwar nur vom Stadium 15/16 Somiten (Embryolänge 1,2-1,8 $\mathrm{mm}$ ).

In dem von mir untersuchten jüngsten Embryo der Spitzmaus dagegen, der sich im Stadium des Kopffortsatzes befand, war das Geschlechtschromatin in den Zellkernen ganz gut ausgesondert, obgleich, die Kernrelation eine solche war, dass man auf ihrer Grundlage das Geschlecht nicht bestimmen konnte. Graham (1954 a, b.) hatte festgestellt, dass von den jüngsten Stadien ab (vor der Gonadendifferenzierung) die Relation der Zellkerne mit Chromatin zu den Chromatinlosen an die erwachsenen Formen angenähert ist. Er bezeichnet es jedoch nicht, von welchem Momente 
ab nach der Befruchtung die Differenzierung der Zellkerne erfolgt. Er behauptet aber jedoch, dass theoretisch genommen, dies schon in der Zygote eintritt.

In der für mich zugänglichen Literatur also in der Arbeit von A ustin und Amorozo (1957) ist festgestellt worden, dass es sich bei der Katze in der zweiten Woche der Schwangerschaft unterscheiden lässt. P a r k e s (1926-27) stellte fest, dass im menschlichen Embryo von \pm 16 Tagen das Geschlechtschromatin sich ebenfalls erblicken lässt. Die erwähnten Autoren geben in ihren Arbeiten jedoch nicht an, ob und von welchem Momente ab bei Fetus die Kernrelation mit Geschlechtschromatin im Vergleich mit denjeningen, die sie nicht besitzen, eine solche ist, dass sie die Altersbestimmung nicht zulässt.

Der Ubergang von einer indifferenten Periode zu einer Differenzierten vollzieht sich, wie es scheint, sehr schnell. In jedem Falle besteht eine plus, minus deutliche Grenze (1,8 mm Länge) oberhalb deren die Kernrelation mit Geschlechtschromatin im Vergleich zu den Chromatinlosen schon eine solche ist, dass sie die fehlerfreie Geschlechtsbestimmung ermöglicht.Das sind zwar nicht so grosse Zahlenunterschiede wie bei älteren Embryonen aber dennoch solche, die keinen Zweifel aufkommen lassen.

Es wirft sich die Frage auf, was für ein Mechanismus es ist? Darauf fällt es schwer zu antworten. Es scheint mir z. B., dass dies auf dem Wege der fortschreitenden Teilung der mitotischen Zellen entstehen könnte, wenn man annimmt, dass bei Männchen sich hier Mitosen der Geschlechtschromatin enthaltenden Zellen verspäten.

Es scheint mir, dass darauf in einem gewissen Grade die mit dem Alter der Embryonen anwachsende Regelung dieser Verhältnisse hinweist. Ihr Prozess erfolgt in der Wachstumsperiode des Embryos von Länge $1,8 \mathrm{~mm}$ bis $3 \mathrm{~mm}$. Embryonen von über $3 \mathrm{~mm}$ Länge haben nämlich schon ungefähr ein solches Verhältnis der Zellkerne mit Geschlechtschromatin zu denjenigen, die es nicht besitzen, wie man es bei erwachsenen Individuen antrifft.

In meiner Arbeit habe ich erwiesen, dass in den jüngsten Würfen das Geschlechtsverhältnis von dem Zustande 1:1 abweicht. Es besteht eine deutliche Überlegenheit der Männchen in allen Monaten ausser dem August. Der physiologische Zustand der Weibchen im August ändert sich unter dem Einfluss irgendwelcher, 
bisher näher unbekannten Faktoren, die auf die Regelung der Gechlechtsverhältnisse im Momente der Befruchtung einen Einfluss ausüben können. In der Literatur (P a r kes, 1926/27) traf ich auf eine sehr grosse Anzahl von hypothetischen Feststellungen über Faktoren, die die zahlenmässigen Geschlechtsverhältnisse regeln können. Es sind: Spermatozoen, Morphologie, Inzucht, Alter der Eltern, Anzahl der durch das Weibchen durchstandenen Schwangerschaften und schliesslich die Ernährung, der man den grössten Einfluss auf die Bildung dieser Verhältnisse zuschreibt.

Wir haben also viele Faktoren, von denen man spricht, dass sie auf den Mechanismus der Geschlechtsbildung unter den Fetus: einen Einfluss ausüben können. Es ist schwer zu sagen, welcher Faktor hier die entscheidende Rolle spielt und ob überhaupt einer von ihnen solche Rolle spielt.

Wenn wir jedoch in dieser Erscheinung irgendeinen biologischen Sinn für die Art erblicken würden - dann müssten wir annehmen das hier vielmehr äusserliche Faktoren wirken müssen und in erster Linie die Ernährung.

Wir wissen es, dass in der Periode des Fetuslebens das Geschlechtsverhältnis sich etwas verändert und dass es bei der Geburt: etwas von dem Zustand abweicht, der im Momente der Befruchtung bestand. In diesem Falle spielt die Fetussterblichkeit die Rolle des Regelungsfaktors.

Nach der Geburt und nach dem Verlassen des Nestes verändert sich das Geschlechtsverhältnis wiederum. Dies hat man auf dem Material junger Spitzmäuse nachgewiesen (B or owski \& Dehnel, Pucek), wobei sich diese Verhältnisse unter den jungen Individuen in verschiedenen Jahren und in Abhängigkeit von den Jahreszeiten verschieden gestalten. Bei den Zusammenstellungen nahm man nur das junge Material in Betracht, so, dass hier keine Störungen im Fangrythmus von physiologischer Natur eine Rolle spielen konnten. Diese Veränderungen in den Geschlechtsverhältnissen kann man nur dem Einfluss der Sterblichkeit zuschreiben. Man kann annehmen, dass es ihrer zweierlei Arten gibt: Die Sterblichkeit im Nest und die Sterblichkeit ausserhalb des Nestes. Es ist eine konkrete Tatsache, dass das zählenmässige Verhältnis des Geschlechtes bei den Spitzmäusen sich innerhalb des Lebens einer Population verändert und in den verschiedenen Sommermonaten verschieden ist. Nichtsdestoweniger ist das Geschlechtsverhältnis unter jungen Individuen aus der Zeitspanne von 10 Jahren (P u - 
c e k, 1959) mit demjenigen, das unter Fetus herrscht, übereinstimmend. In beiden Fällen gibt es eine UUberlegenheit an Männchen. Interessant ist, wenn auch vielleicht zufällig, die Konvergenz der Angaben aus den zwei Monaten, Juni und August bei Jungen (P u c e k). Im Juni haben wir eine Uberzahl an Männchen, im August dagegen in beiden Fällen eine Uberzahl an Weibchen.

Die Ergebnisse meiner Arbeit bestätigten ebenfalls in einem gewissen Grade die Ansichten von Parkes, dass die Anzahl der Würfe keinen Einfluss auf die Gestaltung des Geschlechtverhältnisse hat ${ }^{3}$ ).

\section{FOLGERUNGEN}

1. Das Fetusgeschlecht lässt sich bei der Spitzmaus auf eine bequeme Weise vermittels der cytologischen Methode in der Grenzen von $1,8 \mathrm{~mm}$ Körperlänge des Embryos bis zu seiner Geburt feststellen, was durch die histologische Methode bestätigt wurde.

2. Die Anzahl der Zelkerne mit Geschlechtschromatin beträgt bei einer allgemeinen Anzahl der Zellkerne Hundert - 33 bei Weibchen und 7 bei Männchen.

3) Während der Vorbereitung meiner Arbeit zum Druck, führte ich meine Untersuchungen an den in der Zeit von 5. VII. bis zum 16. IX. 1959 gefangenen trächtigen Weibchen weiter. Das Material bestand aus 29 Embryonen, die von 7 Weibchen stammten. Ich gab darauf acht, dass das Material frisch war und dass die Embryonen gleich nach dem Einbringen nach Zenker fixiert wurden. Die $6 \mu$ Parafinschnitte wurden nach Feulgen gefärbt.

Die jüngsten von mir untersuchten Embryonen waren im Stadium der Kopfverlängerung und die ältesten im Stadium von zirka 20 Somiten.

Das Geschlechtschromatin war schon vom frühesten Stadium ab sichtbar, was jedoch nicht mit der Möglichkeit der Geschlechtsbestimmung gleichwertig ist. In dem jüngsten Wurf, der 4 Embryone umfasste, gelang es mir in zwei Fällen das Geschlecht zu bestimmen, nämlich: Ein Weibchen mit 40 und ein Männchen mit 9 Kernen mit Geschlechtschromatin auf 100. In den beiden übrigen Fällen gab es einmal 13 und ein andermal 12 Kerne mit Geschlechtschromatin auf 100.

In den von mir untersuchten Embryonen schwankte durchschnittlich das Geschlechtschromatin in den Zellkernen auf die gesamte Anzahl von 100 Zellkernen bei Weibchen von 35 bis 61 , bei Männchen von 6 bis 9 . In einem gewissem Falle betrug jedoch die Anzahl des Geschlechtschromatin's auch in diesem neuen Material von 11 bis 18 auf 100 .

Diese Ergebnisse decken sich mit meinen Vorherigen, wo ich festgestellt habe, dass man das Embryogeschlecht im ganzen Wurf nur bei älteren Embryonen als im Stadium von 16 Somiten fehlerfrei bestimmen kann. 
3. Bei kleineren Embryonen als $1,8 \mathrm{~mm}$ konnte man das Geschlecht nicht durch die cytologische Methode bestimmen, denn das Verhältnis der Kerne mit Chromatin zu den Chromatinlosen liegt in der überwiegenden Mehrheit mittelbar zwischen denjenigen Werten, die den angenommenen Zahlen für Männchen und Weibchen entsprechen.

4. Die Regelung dieses Verhältnisses vollzieht sich im Stadium oberhalb von 1,8 $\mathrm{mm}$ Körperlänge d. i. erst bei Embryonen mit über 16 Protosomiten.

5. Bei den Fetus treffen wir auf eine ziemlich bedeutende Überlegenheit der Anzahl der Männchen den Weibchen gegenüber. Nur im August gibt es bei schwangeren Weibchen eine Uberzahl an $\mathrm{Fe}-$ tus des weiblichen Geschlechtes.

6. Es scheint mir, dass in der Periode des Fetuslebens eine grössere Sterblichkeit der Embryonen von männlichem Geschlecht besteht. Darauf weist das Geschlechtsverhältnis in den Würfen hin, bei denen es keine Sterblichkeit gab, im Vergleich zu denjenigen, bei denen eine Sterblichkeit auftrat.

7. Das zahlenmässige Verhältnis der Männchen den Weibchen gegenüber ist von der Embryonenanzahl in der Würfen unabhängig.

Ich erlaube mir Herrn Prof. Dr. August De hnel und Herrn Dr. Andrzej K. Tarkowski für die wertvollen Ratschläge und Fingerzeige, an denen es nicht fehlte, während der Ausführung und schriftlichen Niederlegung vorliegender Arbeit, meinen herzlichsten Dank auszusagen.

Institut für Säugetierforschung

in Białowieża,

Polnische Akademie der Wissenschaften.

\section{SCHRIFTTUM}

1. Austin, C. R. \& Amorozo, E. C. - Sex chromatin in early cat embryos. Exp. Cell. Res., Vol. 13:419-421. London, 1957.

2. Barr, M. L. - The sex chromatin and its application to errors in sex development. ( $\mathrm{I}_{\mathrm{n}}$,Modern Trends in Obstetrics and Gynaccology”, ed. by Kenneth B ow 9 s), 117-128. Butterworth Co. London, 1957.

3. Borowski, S. \& Dehnel, A. - Materialy do biologii Soricide. Ann. Univ. M. Curie-Skłodowska, Sect. C, Vol. 7, 6:305-421. Lublin, 1957.

4. Boy d, T. D. \& Hamilton, W. J. - The cellular components of the human ovary. (In "Modern Trends in Obstetrics and Gynaecology”, ed. by Kenneth Bowes). 50-60. Butterwortb Co. London, 1957. 
5. Brambeil, F. W. R. - Ovarian changes. (In „Marshall's Physiology of Reproduction, ed. by A. S. P a rkes). Vol. 1:397-408. Longmans Green and Co. London, 1955.

6. Godlewski, E. - Embriologia zwierząt kręgowych. Państwowy Zakład Wydawnictw Lekarskich. Warszawa, 1956.

7. Graham, MI. A. - Determination of the sex of cat embryos from nuclear morphology in the embryonic membrane. Nature. Vol. 173:310. London, 1951a.

8. Graham, M. A. - Sex chromatin in cell nuclei of the cat from the early embryo to maturity. Anat. Rec., Vol. 4:469. Philadelphia, 1954b.

9. M oore, K. L. \& B a r r, M. L. - Morphology of the nerve cell nucleus in mammals with special reference to the sex chromatin. J. comp. Neurol., Vol. 98: 213-231. 1953. (Citet acc. to B a r r, 1957).

10. Parkes, A. S. - The mammalian sex ratio. Biol. Rev., Vol. 2:1-51. Cambridge, 1926-27.

11. Pucek, Z. - Some biological aspects of the sex-ratio in the Common Shrew (Sorex araneus araneus L.). Acta Theriol., Vol. 3:43-73. Białowieża, 1959.

12. Ta rkowski, A. K. - Badania nad rozrodem i śmiertelnością zarodkową u ryjówki aksamitnej (Sorex araneus araneus L.). Część II - Rozrod w warunkach naturalnych. Ann. Univ. M. Curie-Skłodowska, Sect. C, Vol. 10:177-229. Lublin, 1955 .

\section{TAFELBFSCHREIBUNG}

Phot. 1. Querschnitt durch den Hoden des Embryos von $7 \mathrm{~mm}$ Länge. Hämatoxylin. Vergr. z-a $200 \times$.

Phot. 2. Querschnitt durch den Eierstock eines Embryos von $8 \mathrm{~mm}$ Länge. Hämatoxylin. Vergr. z-a $200 \times$.

Phot. 3. Querschnitt durch den Eierstock eines Embryos von $13 \mathrm{~mm}$ Länge. Vergr. z-a $200 \times$.

Phot. 4. Geschlechtschromatin in den Zellkernen aus einem Querschnitt durch die Extremität des Embryos (Nr. 4371, P 2) von $8,8 \mathrm{~mm}$ Länge Hämatoxylin. Vergr. z-a $1600 \times$.

\section{STRESZCZENIE}

1. Płeć płodów ryjówiki daje się w sposób dogodny oznaczać metodą cytologiczną w zakresie od $1,8 \mathrm{~mm}$ długości ciała zarodka, aż do urođzenia, co zostało potwierdzone metodą histologiezną.

2. Ilość jąder komórkowych z chromatyną pỉciową na ogölną ilość stu jąder wynosi u samir 33, a u samców 7 (Tabela 3).

3. U zarodków mniejszych niż $1,8 \mathrm{~mm}$ nie można oznaczyć płci metodą cytologiczną, gdyż stosunek jąder z chromatyną płciową układa się w przeważającej większości pośrednio pomiędzy wartościami odpowiadającymi przyjętym liczbom dla samców i samic. 
4. Regulacja tego stnsunku zachodzi w stadium powyżej $1,8 \mathrm{~mm}$ dług ości i ciała tj. dopiero u zarodków powyżej 16 par protosomitów.

5. U płodów spotykamy się z dość znaczną przewagą ilości samców nad samicami. Jedynie w sierpniu obserwuje się przewagę płodów płci żeńsłkiej j (Tabela 4).

6. Wydaje się, że w okresie życia płodowego istnieje większa śmiertelność embrionow płei męskiej. Wskazuje na to stosunek płci w miotach, w których I nie było śmiertelności i u których śmiertelność wystąpiła (Tabela 5).

7. Stosunek ilościowy samic do samców nie jest zależny od ilości embrio- nów w miocie (Tabela 6).

\footnotetext{
Państwowe Wydawnictwo Naukowe * Warszawa 1959 r. Nakład 1455 egz. Ark. wyd. 1,348. Maszyn. otrzym. 5. X. 1959 r. Podpisano do druku 12. XII. $1959 \mathrm{r}$. Druk ukończ. 23. XII. $1959 \mathrm{r}$. Papier druk. sat. kl. II 80 g. Format B5

Białostockie Zakłady Graficzne. Zam. 2824. Cena 6 zl.
} 
ACTA THERIOLOGICA Vol. III, 9.

Tafel XI.
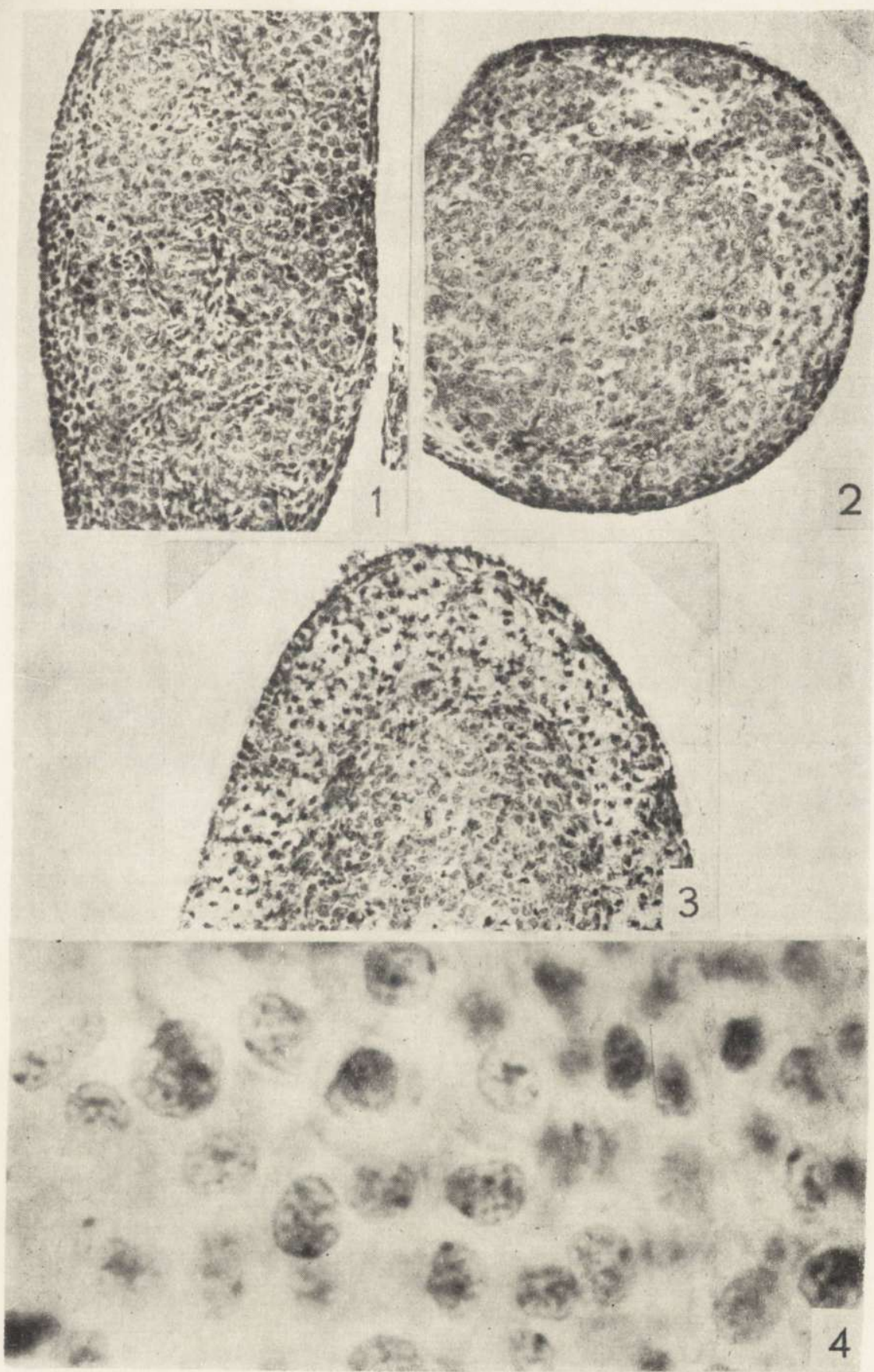

Ewa T. Mystkowska

Z. Pucek \& A. Tarkowski phot. 
BIBLIOTEKA

Instytutu Biologii Ssaków

Polskiej Akademii Nauk

$\mathrm{NrCz} .40 .2$ 